\title{
AFOS: Probing the UV-visible potential of the Antarctic Plateau
}

\author{
J. T. Dempsey ${ }^{a}$, J. W. V. Storey ${ }^{a}$, M. C. B. Ashley ${ }^{a}$, M. G. Burton ${ }^{a}$, P. G. Calisse ${ }^{a}$, M. A. \\ Jarnyk $^{b}$ \\ ${ }^{a}$ School of Physics, University of New South Wales, Sydney NSW Australia 2052; \\ ${ }^{b}$ Research School of Astronomy and Astrophysics, Australian National University, ACT;
}

\begin{abstract}
The Antarctic Fiber-Optic Spectrometer (AFOS) is a $30 \mathrm{~cm}$ Newtonian optical telescope that injects light through six $30 \mathrm{~m}$ long optical fibers onto a $240-850 \mathrm{~nm}$ spectrograph with a $1024 \times 256$ pixel CCD camera. The telescope is mounted on a dual telescope altitude-azimuth mount and has been designed to measure the transperency of the atmosphere above the South Pole for astronomy in the UV and visible wavelength regions. The instrument has observed a series of bright $\mathrm{O}$ and B stars during the austral winters of 2002 and 2003 to probe the UV cutoff wavelength, the auroral intensity and water vapour content in the atmosphere above the plateau.
\end{abstract}

AFOS is the first completely automated optical telescope on the Antarctic Plateau. This paper reports on the results of the past two austral winters of remote observing with the telescope as well as the technical and software modifications required to improve the quality and automation of the observations. The atmospheric absorption bands in the 660-900nm regions of the spectra have been fitted with MODTRAN atmospheric models and used to calculate the precipitable water vapour above the South Pole. These data are then compared to those collected concurrently by radiosonde and by a $350 \mu \mathrm{m}$ submillimeter tipper at South Pole.

Keywords: optical astronomy, fibre optics, Antarctica

\section{INTRODUCTION}

Antarctic instrumentation poses two sets of challenges to the designers and observers. It requires specialised design for the extreme cold-temperatures that are otherwise only experienced by space-based instruments. In addition to working at temperatures averaging $-60^{\circ} \mathrm{C}$ during the winter, Antarctic instruments must either prevent or withstand the formation of ice on apertures, windows and moving parts. Finally these instruments must be well-tested so that they require minimum maintenance during the austral winter.

The AFOS, as seen in Figure 1, is a pioneering telescope in this area. A number of sites on the high antarctic plateau promise excellent observing conditions but do not have the necessary infrastructure to support habitation for any extended length of time. Low-maintenance, low-power instrumentation which requires little or no manual intervention for extended periods is essential for such sites. Achieving successful operation of such a telescope also demonstrates the feasibility of Antarctic sites for large-scale telescope projects.

The AFOS was deployed to Amundsen-Scott base on a dual-telescope alt-az mount. A data set of spectra were collected of the June 2000 total lunar eclipse. The winter observing seasons of 2002 and 2003 allowed extensive testing of the mechanical systems, while the mechanical flexure and pointing accuracy of the mount and tower were characterised to optimise the pointing for automated observations.

Further author information: (Send correspondence to J.T.Dempsey)

J.T.D.: E-mail: jtd@phys.unsw.edu.au, Telephone: 61293854560 


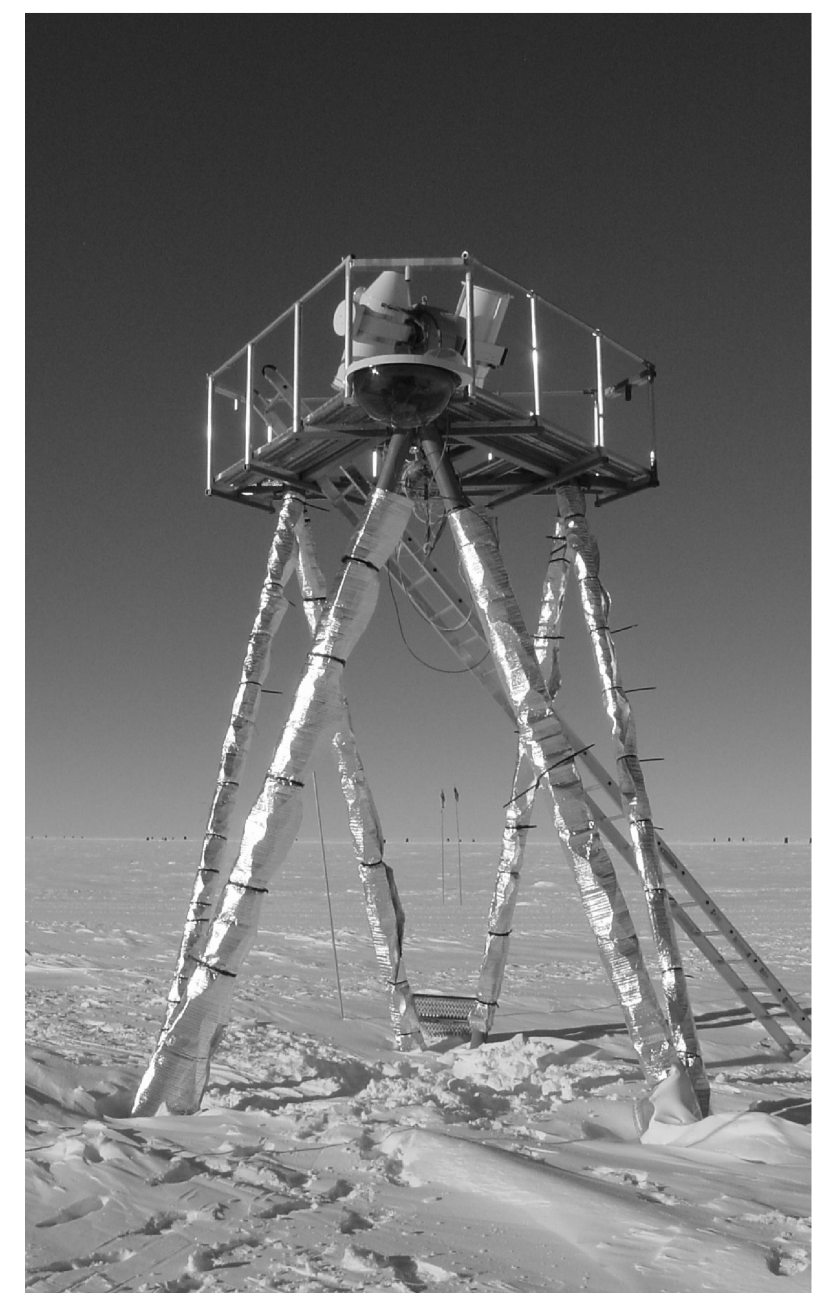

Figure 1. The AFOS telescope (on the right) installed on the G-mount, January 2002.

\section{INSTRUMENT DESCRIPTION}

\subsection{Telescope construction}

The design and construction of the AFOS are detailed in Boccas et.al. ${ }^{1}$ A Newtonian optics system was chosen to minimise complexity. Invar 36, with an expansion coefficient of $\alpha=0.9 \times 10^{-6} K^{-1}$, was used in the construction of all the mechanical parts, while the primary parabolic mirror was formed from Astrosittal, a low thermal expansion glass $\left(\alpha=3.2 \times 10^{-6} K^{-1}\right)$. These selected materials provide sufficient athermalisation for the AFOS to require no mechanical adjustments for aligning mirrors or focussing at both potential test sites in Australia and also in the sub $-80^{\circ} \mathrm{C}$ Antarctic winter.

The primary mirror has a diameter of $318 \mathrm{~mm}$ while the secondary is an elliptical flat mirror with a major axis of $108 \mathrm{~mm}$. Both were given UV-enhanced coatings of $\mathrm{AlMgF}_{2}$ to yield an $85 \%$ reflectivity from 300 to $850 \mathrm{~nm}$. The primary has a focal ratio of $\mathrm{f} / 3.35$ which is focussed into an Invar injection module. A dichroic beam splitter ( $\mathrm{R} \geq 50 \%$ from $300-550 \mathrm{~nm}$, and $\mathrm{T} \geq 50 \%$ from $550-850 \mathrm{~nm}$ ), directs the light into two fibre bundles, one optimised in the red and the other in the blue region of the spectrum to maximise the performance of the fibres across the range of the visible wavelength band. This beamsplitter also performs the order-sorting for the grating spectrome- 


\subsection{Fibre Optics}

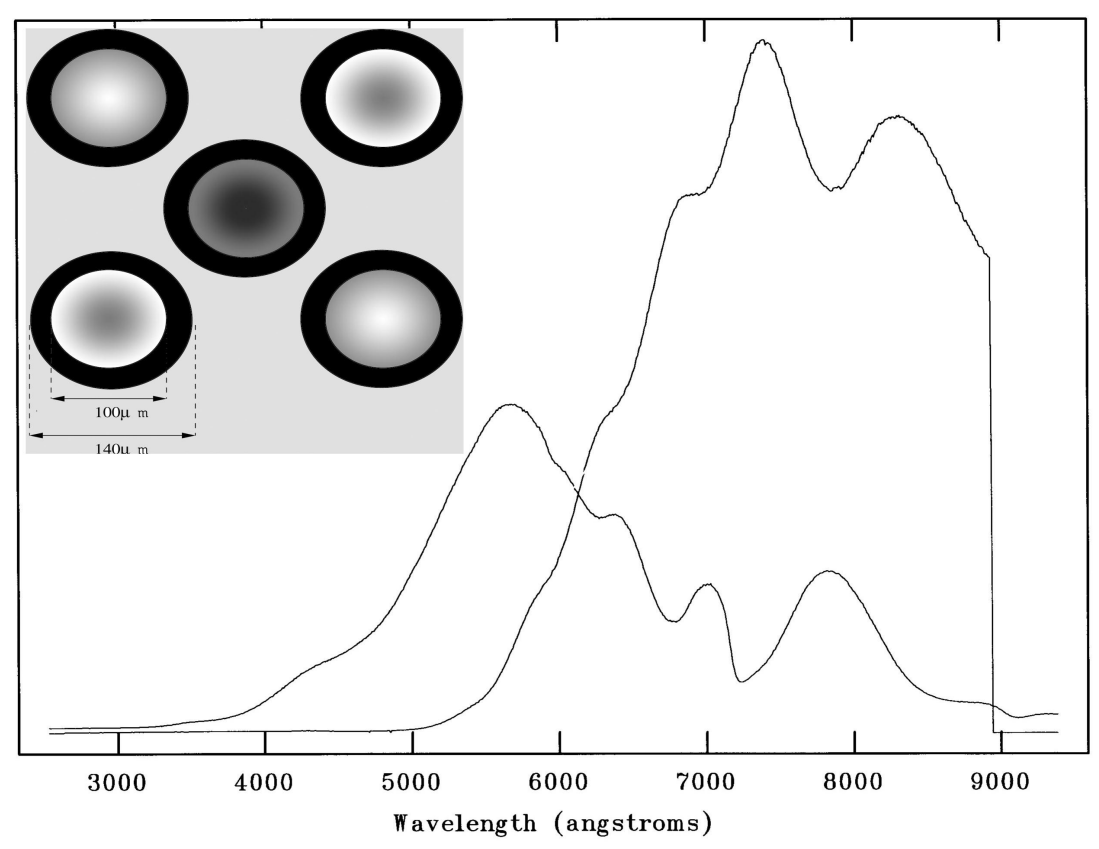

Figure 2. Quartz-halogen lamp response through beamsplitter in red and blue fibres showing the reflectance and transmittance of the beamsplitter in the AFOS. The Quartz-halogen lamp is at a temperature of approximately 3100K. INSET: $\mathrm{X}$-shaped configuration of the fibres on the sky. The central blue(dark) and red(light) fibres overlap the same position on the sky.

Fibre optics are a favoured and now common tool in astronomical spectroscopy. ${ }^{2}$ Particularly for multi-object and wide-field applications, fibre optic transmission provides versatility and stability. ${ }^{3}$ In the particular case of the AFOS, fibre optic transmission provides us with the ability to house the delicate and expensive detection system consisting of a spectrograph and a $1024 \times 256$ pixel CCD camera within the protected environment of the AASTO, while the telescope could be positioned up to 40 meters away, on a 7 meter tower, to avoid local thermal emissions and ground turbulence.

The AFOS was required for observing deep into the blue and UV bands, but observations of atmospheric absorption required reasonable transmission in the red wavelengths as well. One set of three fibres was selected

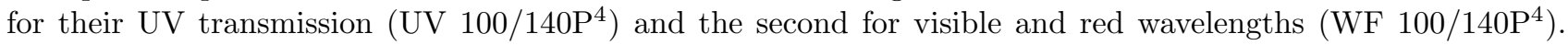
The combined spectral response of the fibres and beamsplitter is shown in Figure 2.

The dichroic beam-splitter injects light into the two sets of fibres, the red and blue optimised bundles, each consisting of three fibres with $100 \mu \mathrm{m}$ diameter fibre cores. $100 \mu \mathrm{m}$ core diameter fibres were selected as a compromise between a small fibre, for high spectroscopic resolution, and a large fibre, to maximise the field of view on the sky, to make target acquisition easier. Each $100 \mu$ m diameter fibre subtends a 19.1 arcsecond field of view on the sky.

To increase the ease of subtracting sky brightness, separate fibres were provided for the observation of the star and the sky background. Both the blue and red fibre bundles consist of three fibres, one to collect the light 
from the star and two bracketing the 'star' fibre, to measure the sky background. The bundles are aligned and set at $90^{\circ}$ to each other in an X-shaped configuration as depicted in Figure 2. The blue and red 'star' fibres are positioned so that they overlap the same position on the sky, to collect the stellar flux simultaneously.

\subsection{Spectrograph and CCD}

The six fibres are aligned single-file in a grooved holder which creates a pseudo-slit for the spectrograph. A commercial imaging spectrograph from Jobin Yvon $\left(1989\right.$, model CP200 ${ }^{5}$ ) was selected. This model has a fixed concave holographic grating of $200 \mathrm{~g} \mathrm{~mm}^{-1}$, and was chosen for its stability and excellent stray light rejection ratio of 1 part in $10^{4}$.

One problem that can be caused by optical fibres is a speeding up of the beam, and this phenomenon is called focal ratio degradation. ${ }^{6}$ The entrance pupil of the spectrograph was matched to the calculated focal ratio that would exit the fibres given the measured focal ratio degredation, which is $\mathrm{f} / 2.9$. The spectrograph focal length is $190 \mathrm{~mm}$ and creates a flat image of $25 \times 8 \mathrm{~mm}$. The spectrograph was required to have optimum possible transmission in the UV, and resolution good enough to be limited only by the pixel size of the CCD array. The resolution of the spectrograph is $2.4 \mathrm{~nm}$ per pixel.

The CCD camera, and controlling software, is an Instaspec IV (Oriel Instruments, 1995 ${ }^{7}$ ). The area of the $\mathrm{CCD}$ is $1024 \times 256$ pixels, each pixel is $27 \times 27 \mu \mathrm{m}$ in area, thus having a total active area of $27.6 \times 6.9 \mathrm{~mm}$. The CCD output is digitised to 16 bits.

\subsection{Generic Mount}

The G-mount was designed and built by Gary Hovey, Ralph Sutherland, Mark Jarnyk and their team at the Research School of Astronomy and Astrophysics (RSAA) at Mt Stromlo Observatory. Designed for extremely low-power $(<50 \mathrm{~W})$ and high precision, the G-mount is a dual-telescope altitude-azimuth mount that simultaneously cradles the AFOS and the Antarctic Differential Image Motion Monitor (ADIMM). ${ }^{8}$ First deployed during the summer of $1999 / 2000$, the mount and its two telescopes were assembled at South Pole and placed on a $7 \mathrm{~m}$ tower approximately 10 metres from the AASTO building.

\section{TESTING AND MODIFICATION}

The AFOS has no pointing camera to improve the ease of centering the telescope on a star. The companion instrument ADIMM can be seen to the left of the AFOS in Figure 1. The ADIMM had a wide-field camera and an automated pointing routine that had been well-tested with the G-mount prior to deployment. Once a pointing sequence was undertaken by the ADIMM and new pointing model entered into the mount's software, a single, fixed offset in altitude and azimuth should center the star in the AFOS aperture. Analysis revealed that polarisation effects caused attenuation in the spectra when the star was not within 5 arcseconds of the centre of the fibre. The pointing runs needed to be more frequent to guarantee this accuracy and so often the telescope position would have to be manually adjusted to improve the star's position.

Manual shifts in the pointing were required to center each star before each observation. While this process was time-consuming, this greatly improved the quality of the spectra and numerous suitable measurements were taken. A thorough instrument analysis was undertaken at the very beginning of the 2003 winter season, and the results of these tests are presented here. The observing and software modifications that resulted allowed a very high quality and quantity of spectra to be taken through the 2003 winter. 


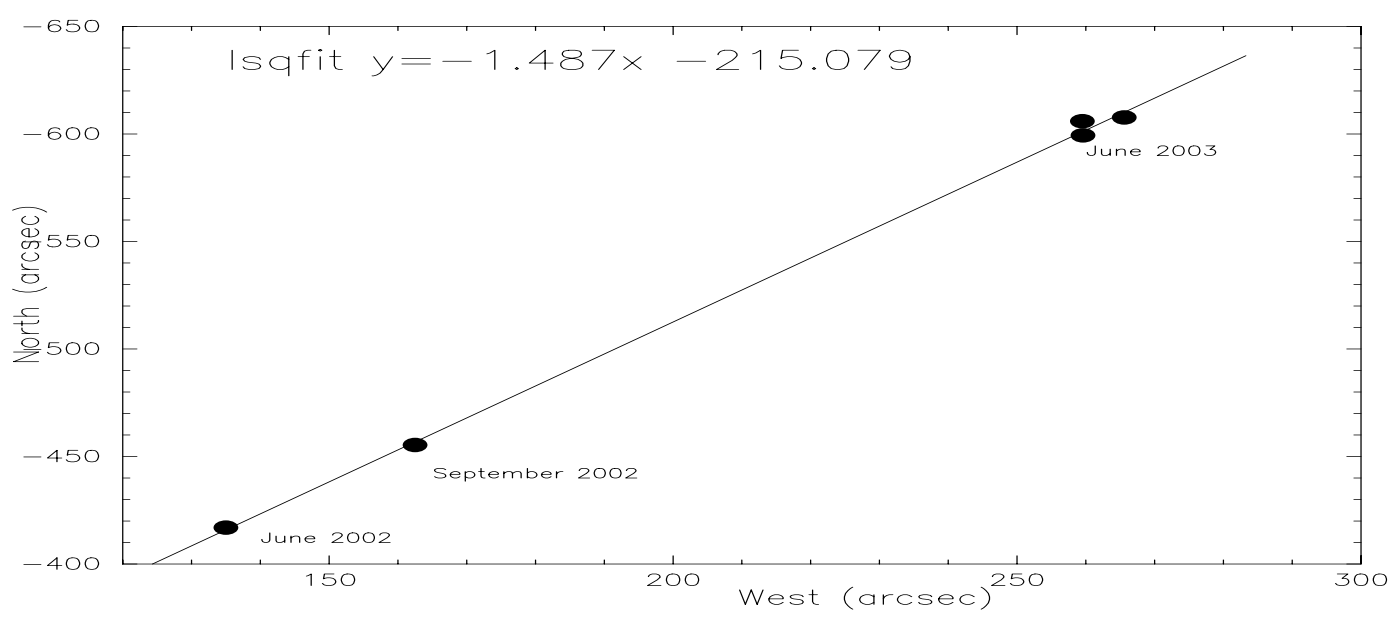

Figure 3. Drift of centre of pointing towards the telescopes north and west axis as a function of time .

\subsection{Tower tilt and sinking}

The ADIMM telescope was programmed with an extensive 300 star pointing program and prior to 2003, Mt Stromlo Observatory ran the data collection and pointing programs. The pointing software was run only three times during 2002, being time-consuming both in telescope time and for the person analysing the run. The program T-point was used to calculate the telescope pointing model. Once this pointing model was logged into the mount software, a single fixed offset in azimuth (approx 600 arcseconds) and altitude (approx 300 arcseconds) was entered to shift the pointing of the focal plane from the ADIMM to the AFOS.

The pointing model collected in June 2003 showed that the tower was sinking towards one particular leg. The plot of this trend is displayed in Figure 3. The 'west' and 'north' indicated in the plot are constructs of the pointing program. It was reasonably assumed in 2002 that a pointing run every month or so should be sufficient and in fact it was adequate for the large $(10 \times 3$ arcminute) field of view camera on the ADIMM. For the 19 arcsecond field-of-view on the AFOS, a tilt amounting to approximately 5 arcseconds of offset per week was significant. Once this problem was identified the number of pointing runs was increased to twice a week.

The improvements in the pointing and observing schedule allowed for an increase in the automation of the observations. In August and September of 2003, a series of 24-hour automated observations of the dark-side of the moon were successfully completed. The satellite connectivity to South Pole only allowed six to eight hours of direct communication with the telescope so it was necessary to construct scripts to run the telescope and data collection during the hours of satellite down-time.

The scripts to run these observations were programmed in TCL language EXPECT. These programs were then further modified for the specific designs of the project, and ideally could be extended to stellar observations allowing for optimisation of the signal and corrections to the pointing during the automated routine.

\section{FITTING SPECTRA USING MODTRAN}

MODTRAN is a MODerate resolution TRANsmission and radiative transfer atmospheric modelling code. ${ }^{9}$ It can calculate the emission or transmission of the atmosphere in a requested wavelength range from the infrared to the optical, once provided with a number of parameters describing the atmospheric conditions. MODTRAN is designed primarily for mid-latitude and temperature atmospheric modelling and is used to predict and confirm both ground-based and satellite atmospheric measurements. It also has an extensive list of user-input options 
that allows the user to model less common atmospheric conditions such as those at South Pole.

\subsection{Radiosonde Profiles}
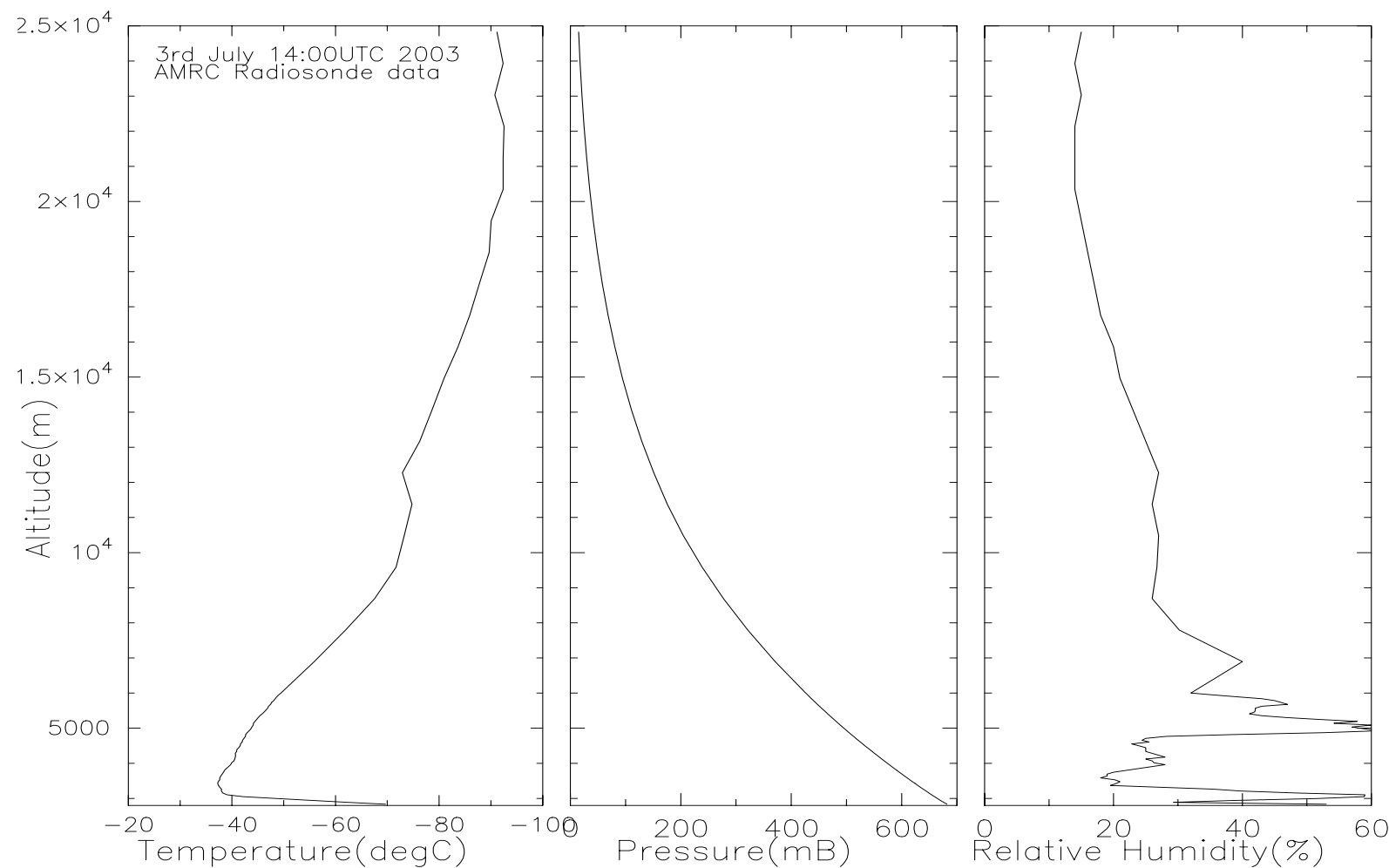

Figure 4. Temperature, pressure and relative humidity profile above South Pole station as a function of altitude, 14:00hrs(UTC), 3rd July 2003. Courtesy of the Antarctic Meteorological Research Centre (AMRC) ${ }^{10}$.

The Antarctic Meteorological Research Center (AMRC) at the University of Wisconsin has launched weather balloons twice-daily at South Pole station since 1956. The radiosonde data for each day of AFOS observations was obtained from their data archive. ${ }^{10}$ The temperature, pressure and relative humidity data from ground-level ( $2836 \mathrm{~m}$ above sea-level) to $26 \mathrm{~km}$ was included in the MODTRAN 'tape 5' input file allowing a model atmosphere to be derived for each day of observation. An example of a typical temperature and wind-speed profile is shown in Figure 4.

In addition to this the MODTRAN file provides options to input the resolution of the spectra, the zenith angle and line-of-sight through the atmosphere required, and the amount of precipitable water vapour, ozone and carbon dioxide to use in the model calculations.

\subsection{Data and Reduction}

On each observing run, the AFOS collected spectra of a series of nine bright $\mathrm{O}$ and $\mathrm{B}$ stars. These stars were selected for their high UV flux and smooth, relatively featureless spectral signatures. As a star does not change in elevation during a 24 hour period at South Pole, the objects needed to be of similar spectral type but at a range of declinations, to allow the atmospheric lines to be observed at different zenith angles. On average, six hours of satellite up-time was available each day for remote observing with the telescope. On a day with good 

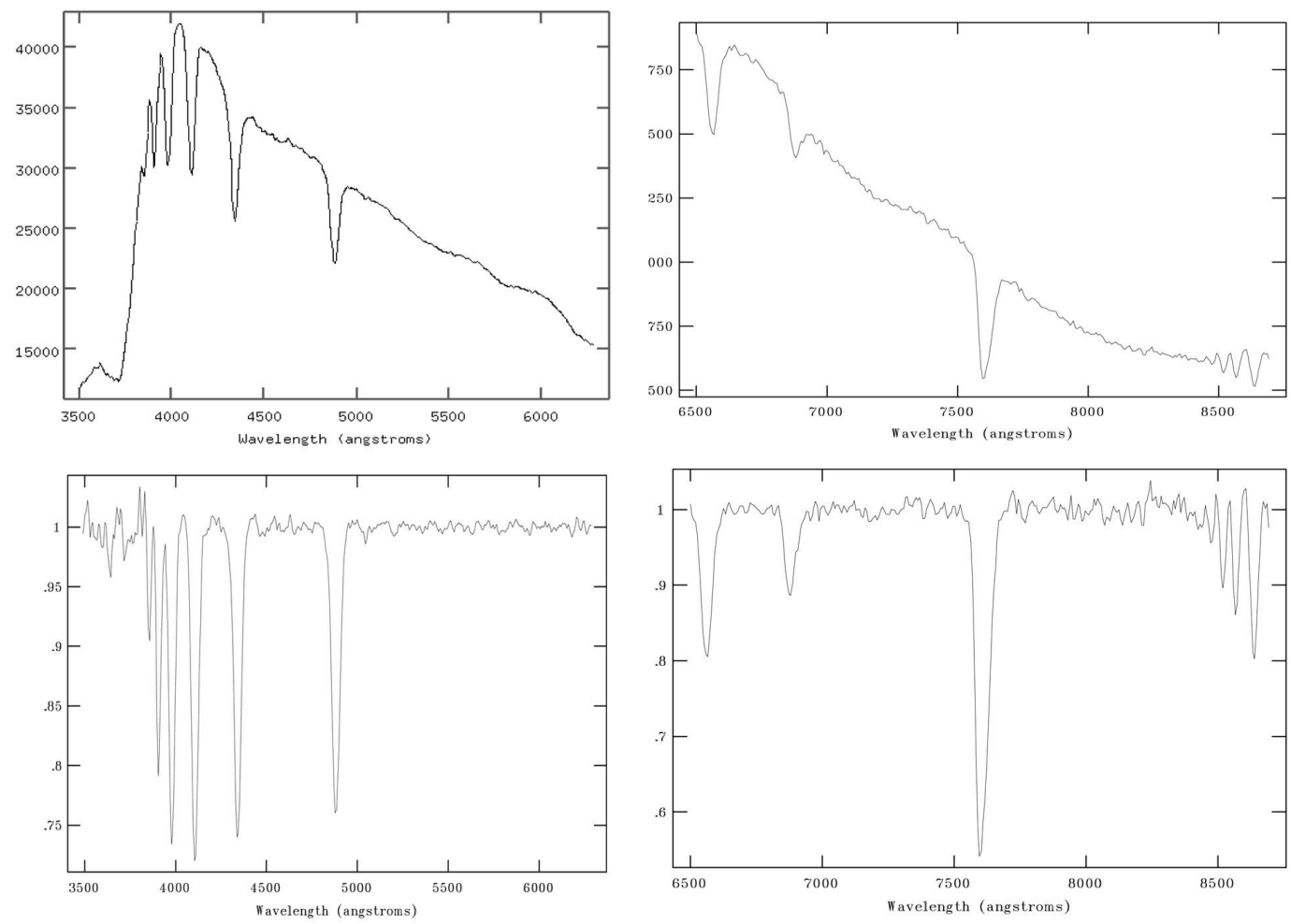

Figure 5. Top Left: Blue fibre spectrum of Beta Centauri, 3rd July 2003. Top Right: Red fibre spectrum of Beta Centauri, 3rd July 2003. Bottom Left: Normalised Blue fibre spectrum of Beta Centauri. Bottom Right: Normalised Red fibre spectrum of Beta Centauri.

conditions and connectivity up to sixty spectra would be collected in this time.

Each CCD image was bias-subtracted and sky-subtracted using IRAF CCD reduction routines. The IRAF package DOFIBRES was then used to extract the fibre apertures from each image. In this way each exposure produces two one-dimensional spectra, one collected in the red-optimised fibre, and a second in the blue. Because of misalignment of the fibre centres two sets of observations needed to be taken each day, optimising for first the blue and then the red aperture.

Once these spectra were obtained, the flat-field spectra of a quartz-halogen lamp within the telescope barrel were subjected to the same reduction as the stellar images. Each stellar spectrum is divided by the flat-field spectrum (seen in Figure 2) and then multiplied by an artificial spectrum of a blackbody at 3300K (the temperature of the quartz-halogen lamp), resulting in the final stellar spectra. An example of a fully-reduced red and blue spectrum, before and after normalisation, can be seen in Figure 5.

\subsection{Fitting for $\mathrm{H}_{2} \mathrm{O}$}

A FORTRAN program was writen to compare the observed spectra with the MODTRAN spectra derived from the radiosonde data. Several models were computed while varying a number of parameters and the model with the lowest chi-squared fit to the data was identified. The model was calculated for the zenith angle of each object. To test the validity of the model, the program was run to fit the deep oxygen absorption lines in the $760 \mathrm{~nm} \mathrm{~A}$ band. These lines are convolved into a single line in the AFOS data as a result of its low resolution. Since this line depth and width is independent of the water vapour content, the line was fit using a model that varied as a function of ground temperature. The resulting best-fit ground temperature for each day (Figure 6) was in good agreement with the ground temperature measured by the radiosondes and the NOAA weather monitors at South 

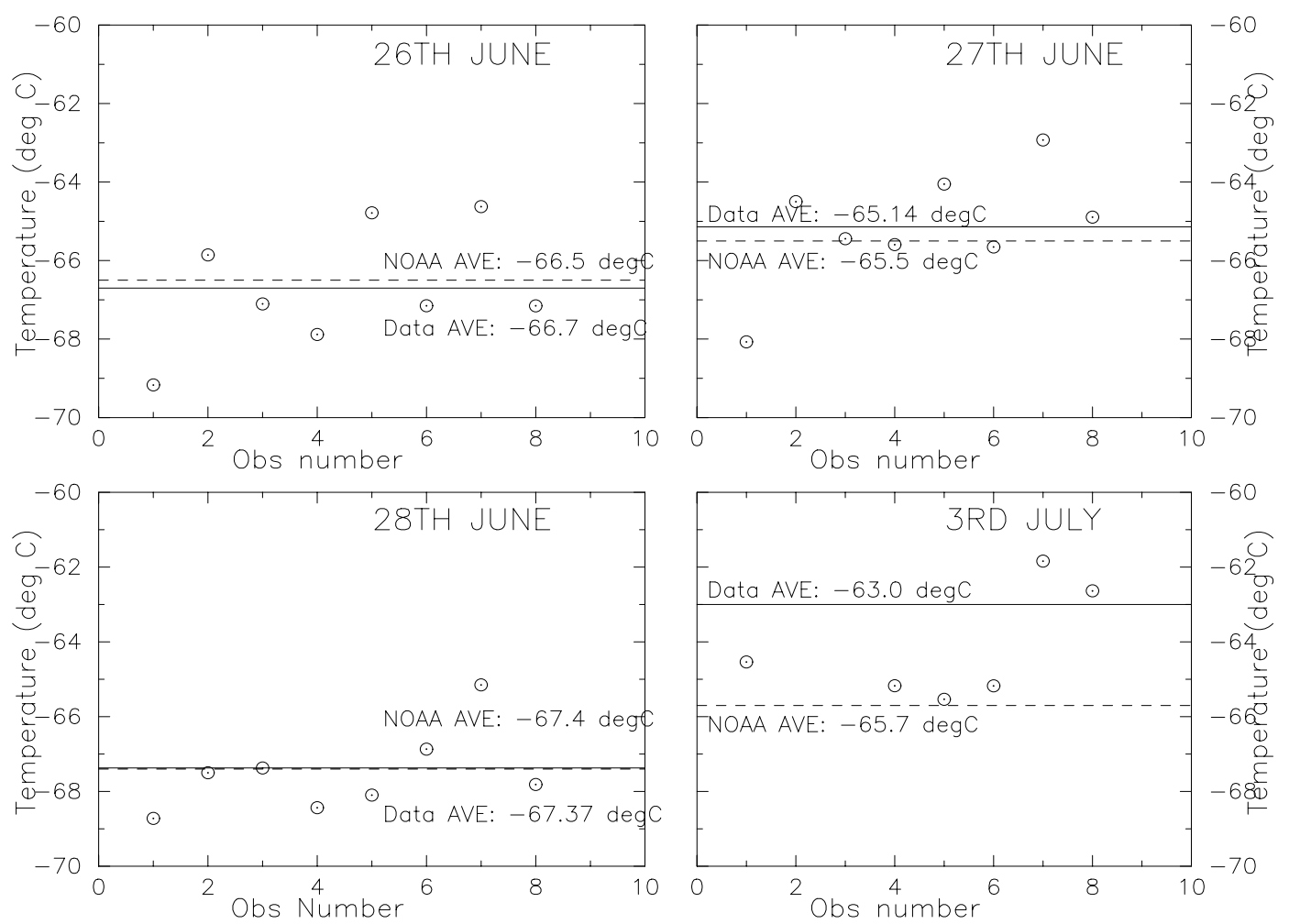

Figure 6. Best-fit ground temperature from the MODTRAN fit to the AFOS data for four separate days in June and July of 2003.

Pole.

The individual MODTRAN files were then fitted to the two water vapour bands in the AFOS spectrum, between $745-755 \mathrm{~nm}$ and $840-855 \mathrm{~nm}$. A sample fit of the model to the data can be seen in Figure 7. The model was generated for a series of $\mathrm{H}_{2} \mathrm{O}$ values and then compared to the data using a chi-squared fit. The output of this program was then plotted and the best fit $\mathrm{H}_{2} \mathrm{O}$ value calculated by minimising the polynomial fit to the results, as shown in Figure 8.

\subsection{Comparison to $350 \mu \mathrm{m}$ Data}

To test whether this method is calculating the $\mathrm{H}_{2} \mathrm{O}$ content accurately, the results were compared with the precipitable water vapour (PWV) content derived from a $350 \mu \mathrm{m}$ submillimeter tipping radiometer located on the AST/RO telescope building at South Pole. The tipper calculates a sky opacity, $\tau$, and from this a PWV content can be derived. ${ }^{11}$ A comparison of the PWV content fitted by the model to the AFOS data was compared directly with the tipper $\tau$ values and are plotted in Figure 9. The trend of this plot shows that the AFOS data can detect variations in the water vapour content on a daily basis, though the accuracy of the fit must be improved, and currently represents an upper-limit on the $\mathrm{H}_{2} \mathrm{O}$ content.

\section{DISCUSSION AND FURTHER WORK}

An improvement in the accuracy of flat-fielding and normalisation of the data will improve the accuracy of the water vapour fits to the AFOS data. With an increase in resolution this technique could be applied to other 


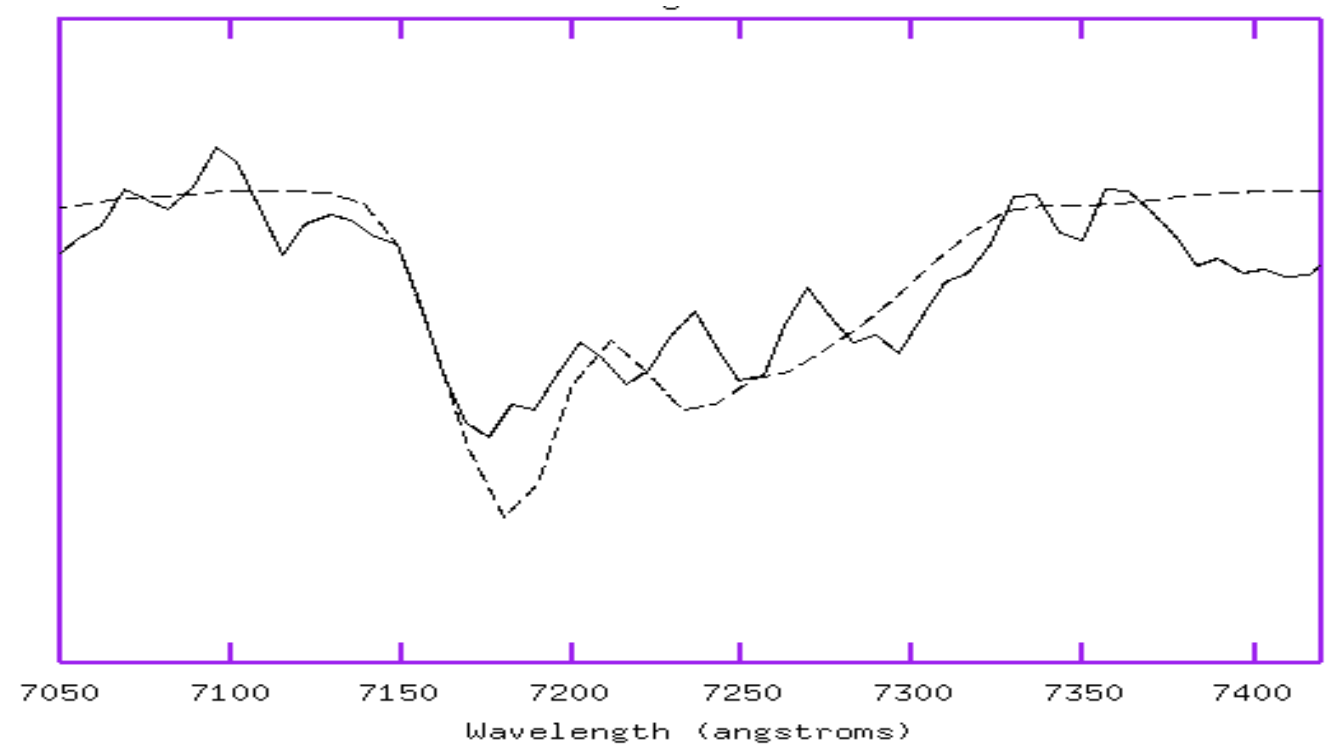

Figure 7. 750nm $\mathrm{H}_{2} \mathrm{O}$ band of Alpha Piscis Austrini compared with a MODTRAN model for the day 27th June 2003.
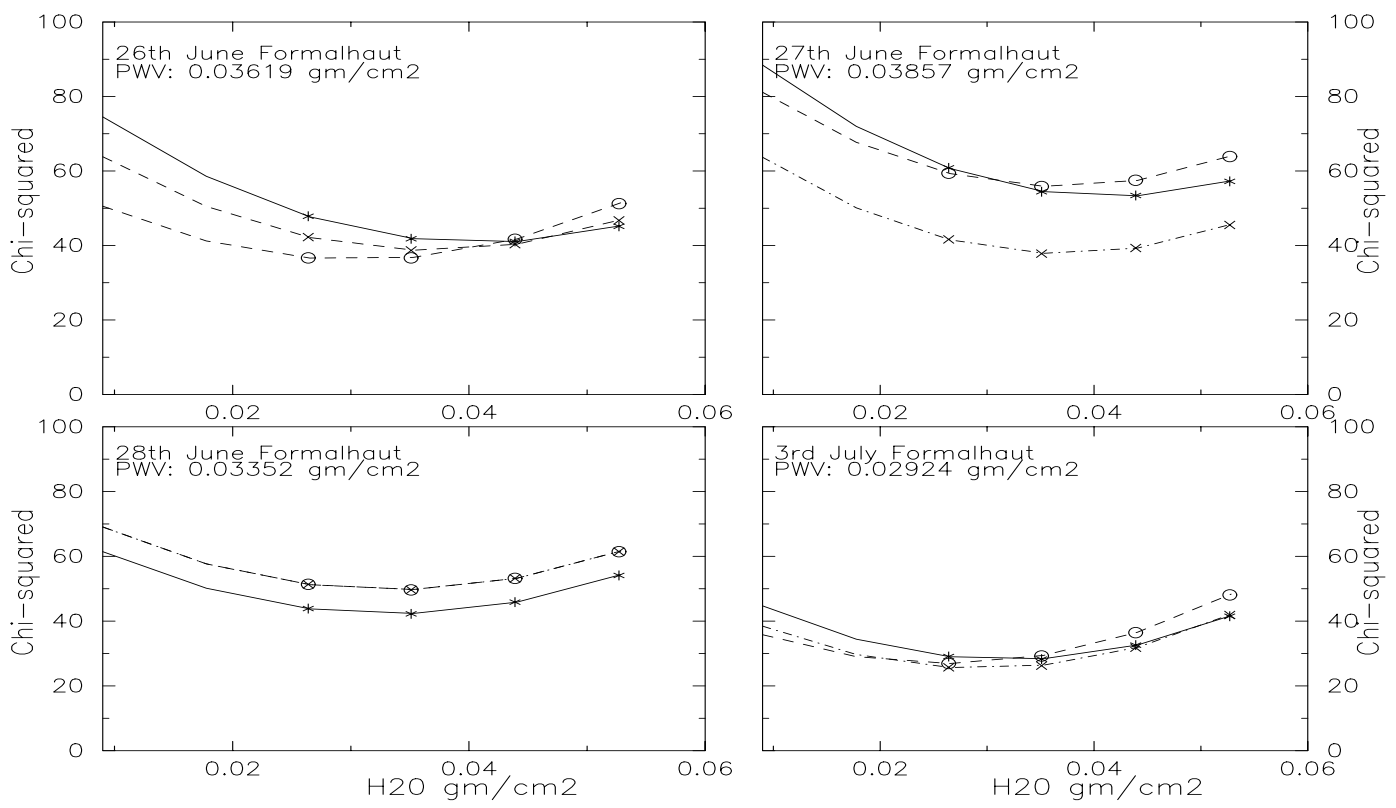

Figure 8. Chi-squared fit to $\mathrm{H}_{2} \mathrm{O}$ band of Alpha Piscis Austrini, 27th June 2003. The three fits are to three spectra of different exposure lengths.

atmospheric constituents, such as ozone. AFOS is the first optical telescope to conduct remote observations during wintertime on the Antarctic Plateau. As the science case for plateau sites like Dome $\mathrm{C}$ grows stronger ${ }^{12},{ }^{13}$ experiments such as AFOS are a useful testbed for large-scale optical astronomy in Antarctica. 


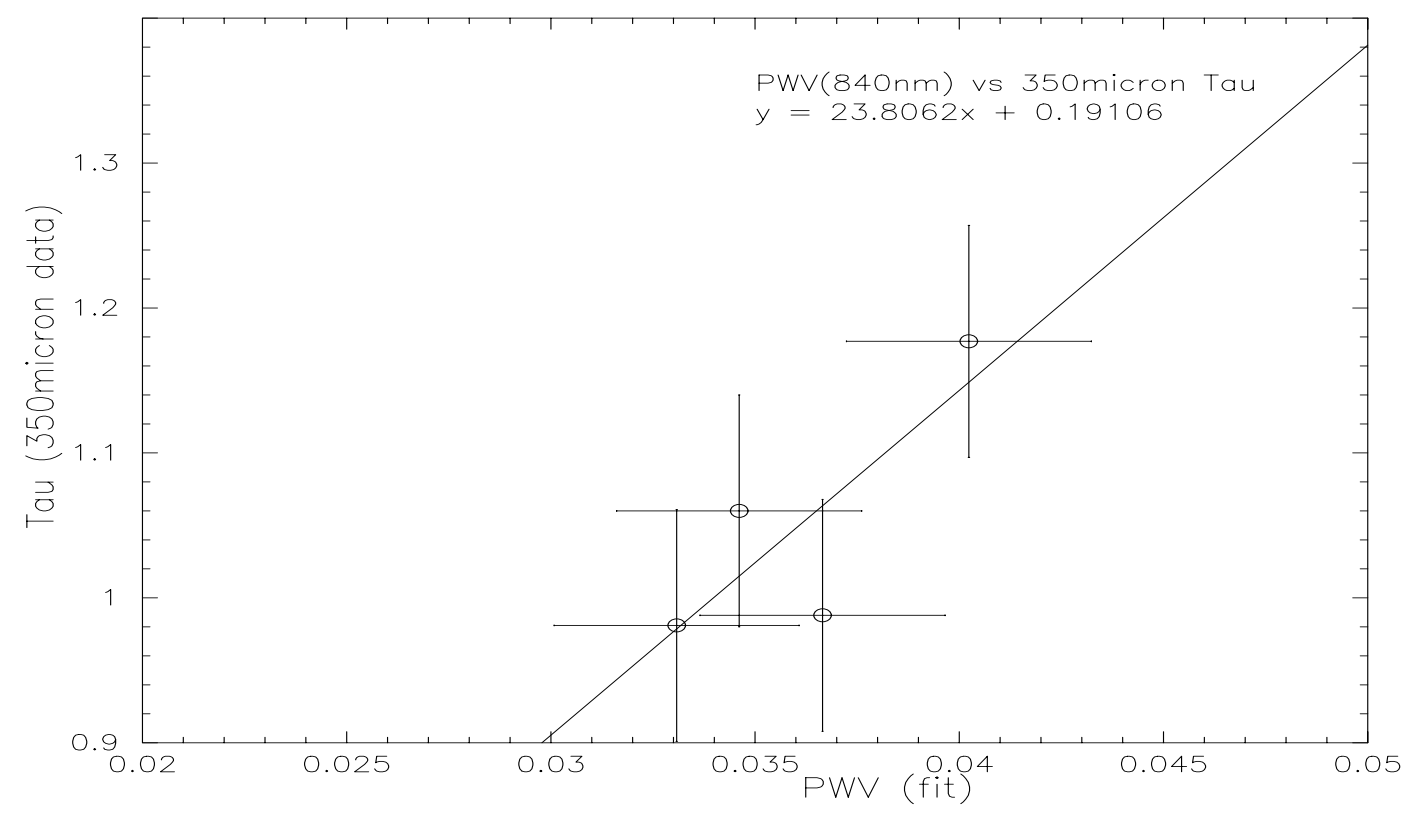

Figure 9. Comparison of $350 \mu \mathrm{m}$ radiometer Sky Opacity $(\tau)$ values with the PWV content $\left(\mathrm{gm} / \mathrm{cm}^{2}\right)$ calculated by the AFOS for four separate days.

\section{ACKNOWLEDGMENTS}

We are grateful to the Antarctic Meteorological Research Centre for providing the radiosonde data used in the atmospheric models. This work was made possible by the National Science Foundation logistical and technical support at South Pole, and by a grant from the Australian Research Council. We are also grateful to Jon Lawrence and Melinda Taylor for helpful discussions and invaluable assistance and to Jon Everett for technical improvements and support.

\section{REFERENCES}

1. M. Boccas, M. C. B. Ashley, A. Phillips, A. Schinckel, and J. W. V. Storey, "Antarctic Fiber Optic Spectrometer," 110, pp. 306-316, Mar. 1998.

2. S. C. Barden, "Review of Fiber-Optic Properties for Astronomical Spectroscopy," in ASP Conf. Ser. 152: Fiber Optics in Astronomy III, pp. 14-+, 1998.

3. D. Vaughnn, "What's wrong with the throughput-resolution product? A fiber-fed spectrograph forces a reevaluation of instrument design parameters," in Proc. SPIE Vol. 2198, p. 31-43, Instrumentation in Astronomy VIII, David L. Crawford; Eric R. Craine; Eds., pp. 31-43, June 1994.

4. CeramOptec Industries Inc., Optran UVWF Fiber Optic Specification Sheet. CramOptec Industries, Inc., East Longmeadow MA 01028, 1 ed., 2003.

5. Jobin Yvon Inc., Guide for Spectroscopy. Jobin Yvon Optics and Spectroscopy Inc., 16-18 Rue Du Canal, Longumeau France, 1 ed., 1993.

6. L. W. Ramsey, "Focal ratio degradation in optical fibers of astronomical interest," in ASP Conf. Ser. 3: Fiber Optics in Astronomy, pp. 26-39, 1988.

7. Oriel Corporation, Instaspec IV CCD Detection Systems Manual. Oriel Corporation, Stratford, CT U.S.A., 1 ed., 1990.

8. M. A. Dopita, P. R. Wood, and G. R. Hovey, "An automated DIMM telescope for Antarctica," Publications of the Astronomical Society of Australia 13, pp. 39-43, Jan. 1996. 
9. A. Berk, L. S. Bernstein, and D. C. Robertson, "MODTRAN: A Moderate Resolution Model for LOWTRAN 7," tech. rep., Air Force Geophysics Laboratory Technical Report, Hanscom AFB, MA., 1993.

10. AMRC, "Antarctic Meteorological Research Center, Data Archives," 2004.

11. R. A. Chamberlin, "South Pole submillimeter sky opacity and correlations with radiosonde observations," pp. 20101-20114, Sept. 2001.

12. R. Angel, J. Lawrence, and J. Storey, "An ELT at Dome C, Antarctica," in Proc. SPIE, Proc. 2nd Backasog Workshop on Extremely Large Telescopes, in press 2003.

13. J. S. Lawrence, M. C. B. Ashley, M. G. Burton, P. G. Calisse, J. T. Dempsey, J. R. Everett, O. Mather, J. W. V. Storey, and T. Travoullion, "The AASTINO: Automated Astrophysical Site Testing International Observatory," in Proceedings of the Conference on Towards Other Earths: DARWIN/TPF and the Search for Extrasolar Terrestrial Planets, 22-25 April 2003, Heidelberg, Germany. Edited by M. Fridlund, T. Henning, compiled by H. Lacoste. ESA SP-539, Noordwijk, Netherlands: ESA Publications Division, pp.497 - 501, 2003. 